\title{
Interpreting Voids in Atom Probe Tomography Data via Experiment and Theory
}

Jonathan D. Poplawsky, ${ }^{1, *}$ Xing Wang, ${ }^{1}$ Constantinos Hatzouglou, ${ }^{2}$ Wei Guo, ${ }^{1}$ Ke Jin, ${ }^{3}$ Hongbin Bei, ${ }^{3}$ Yongqiang Wang, ${ }^{4}$ William J. Weber, ${ }^{3,5}$ Yanwen Zhang, ${ }^{3,5}$ Francois Vurpillot, ${ }^{2}$ and Karren L. More ${ }^{1}$

1. Center for Nanophase Materials Sciences, Oak Ridge National Laboratory, Oak Ridge, TN, USA.

2. Normandie Université, UNIROUEN, INSA Rouen, CNRS, GPM, Rouen, France.

3. Materials Science and Technology Division, Oak Ridge National Laboratory, Oak Ridge, TN, USA.

4. Materials Science and Technology Division, Los Alamos National Laboratory, Los Alamos, NM, USA.

5. Department of Materials Science and Engineering, University of Tennessee, Knoxville, TN, USA.

*Corresponding author: poplawskyjd@ornl.gov

Nanosized voids and/or pores are features that occur in many important materials for energy applications such as zeolites and nuclear materials [1-4]. Quantifying elemental segregation associated with voids at the nm-scale is important for understanding atomic-level material functionality as well as degradation mechanisms. For instance, He bubbles that form in irradiated structural materials cause unwanted swelling and embrittlement and can ultimately lead to failure of these materials $[2,4]$. Understanding elemental segregation effects associated with He bubbles in irradiated alloys is important for determining defect (vacancies and interstitials) diffusion/migration mechanisms, which is important for designing radiation-tolerant alloys. A commonly used method for nanoscale material characterization is electron microscopy; however, the small volume of individual voids with respect to typical scanning/transmission electron microscopy (STEM) lamella thicknesses make compositional quantification of the void shell difficult. The specified local electrode atom probe (LEAP) resolution ranges from $0.1 \mathrm{~nm}$ in $z$ and $0.3 \mathrm{~nm}$ in $x$ and $y$, making this an ideal technique for measuring the local compositions surrounding voids; however, the erratic ion flight paths from the void opening during field evaporation severely compromises the LEAP resolution. Understanding the field evaporation behavior of voids via atom probe tomography (APT) will enable improved methods for interpreting the reconstructed data and for comparing data acquired for different materials.

APT data for several single-phase concentrated solid-solution alloys (SP-CSAs), including NiCo, NiFe, NiCoCr, $\mathrm{NiCoFe}$, and $\mathrm{NiCoCrFe}$, was acquired and then analyzed using the reconstruction method currently available in IVAS that assumes a constant tip diameter for a hemispherically shaped needle. SP-CSAs are ideal specimens for understanding void evaporation behavior because the localized elemental segregation surrounding a small void acts as a marker for identifying the detector hit location of the void shell during field evaporation. For NiCoCr and $\mathrm{NiCoCrFe}$, a correlative STEM/APT approach using the same needle-shaped sample was used to confirm the location of He bubbles in the APT datasets and to determine if He bubble sizes could be extracted from the APT data. Results of the correlative STEM and APT experiment for NiCoCr are shown in Figures $1 \mathrm{a}$ and $1 \mathrm{~b}$, respectively. Voids were consistently identified as high-density regions for $\mathrm{NiCoCr}$ and low-density regions for $\mathrm{NiCoCrFe}$ in the APT data.

Simulated APT experiments of needles containing voids were performed to explain the observed density phenomena, in which simulated detector data was reconstructed using the same method as the experimental APT data. The simulated APT needles contained voids with a $2 \mathrm{~nm}$ elemental segregation shell around the voids. The void size to needle radius was kept constant for the simulations while the evaporation field for the segregation layer around the void was varied with respect to the matrix. Three voids were included in the simulations and included segregation layer evaporation fields that were lower, higher, and the same as the matrix. In the APT reconstruction, the density and concentration profiles of the voids were different for the three different cases studied. The simulation for the high evaporation field case indicated a high-density region for the void, while the simulation for the low evaporation field case contained a low-density region for the void in the APT reconstruction. These high and low evaporation field simulations match the experimental data obtained for $\mathrm{NiCoCr}$ (shown in Figure 1) and $\mathrm{NiCoCrFe}$, respectively. The $\mathrm{Cr}^{++} / \mathrm{Cr}^{+}$charge state ratio within the void segregation region was compared to that of the matrix in the experimental data to determine the field difference between the segregation layer and the matrix. 
The void segregation layer in NiCoCr showed a smaller $\mathrm{Cr}^{++} / \mathrm{Cr}^{+}$ratio than the matrix, whereas the segregation layer around the void in $\mathrm{NiCoCrFe}$ showed a larger $\mathrm{Cr}^{++} / \mathrm{Cr}^{+}$ratio than the matrix, indicating that the segregation layer in $\mathrm{NiCoCr}$ had a lower evaporation field than the matrix and that in $\mathrm{NiCoCrFe}$ had a higher evaporation field than the matrix. The simulated and experimental APT data are in excellent agreement, such that the simulations correctly predicted how the segregation layer around the voids evaporates and can be used to correctly interpret APT data and improve void/bubble reconstructions.

A qualitative model for void evaporation as well as best practices to capture, identify, and measure void sizes, and to quantify and compare segregation behavior around voids in APT reconstructions will be presented. A fundamental understanding of void evaporation processes in multi-element SP-CSAs will lead to more robust analyses of nano-void containing materials by APT [5].

\section{References:}

[1] A Devaraj et al., Scientific Reports 6 (2016), p. 37586.

[2] PD Edmondson et al., Scripta Materialia 65 (2011), p. 731.

[3] J Schmidt et al., Angewandte Chemie International Edition 57 (2018), p. 10422.

[4] MK Miller et al., Ultramicroscopy 111 (2011), p. 469.

[5] This work was supported as part of the Energy Dissipation to Defect Evolution (EDDE), an Energy Frontier Research Center funded by the U.S. Department of Energy (DOE), Office of Science, Basic Energy Sciences under contract number DE-AC05-00OR22725. APT and STEM performed at ORNL's Center for Nanophase Materials Sciences, which is a U.S. DOE, Office of Science User Facility. Helium implantations were supported by the Center for Integrated Nanotechnologies (CINT), a DOE Office of Science User Facility jointly operated by Los Alamos and Sandia National Laboratories. This manuscript has been authored by UT-Battelle, LLC under Contract No. DE-AC05-00OR22725 with the U.S. Department of Energy. The United States Government retains and the publisher, by accepting the article for publication, acknowledges that the United States Government retains a non-exclusive, paid-up, irrevocable, world-wide license to publish or reproduce the published form of this manuscript, or allow others to do so, for United States Government purposes. The Department of Energy will provide public access to these results of federally sponsored research in accordance with the DOE Public Access Plan (http://energy.gov/downloads/doe-public-access-plan).
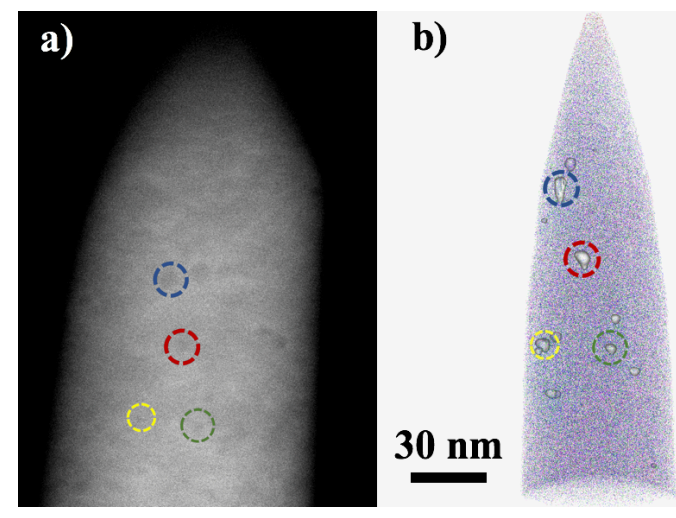

c)

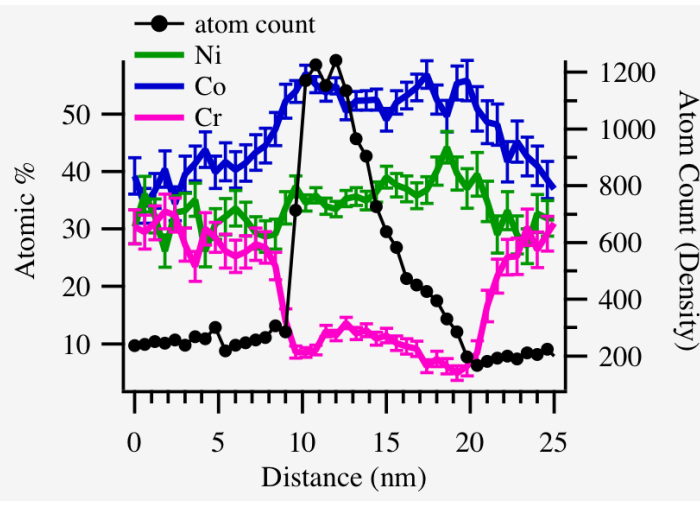

Figure 1. a) HAADF-STEM image and b) APT atom map with iso-density surfaces highlighting high density regions in a He-irradiated $\mathrm{NiCoCr}$ alloy. (c) $1 \mathrm{D}$ line profile in $z$-direction of red-circled void in (b) showing $\mathrm{Cr}$ depletion in void and high-density region on top of void. 